\title{
Sentinel Lymph Node Biopsy Versus Elective Neck Dissection: Long-Term Oncologic Outcomes in Clinically Node-Negative Tongue Cancer
}

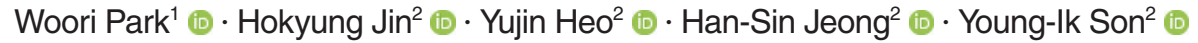 \\ Man Ki Chung ${ }^{2}$ (i) $\cdot$ Chung-Hwan Baek ${ }^{2}$ (i) \\ ${ }^{1}$ Department of Otorhinolaryngology-Head and Neck Surgery, Uijeongbu Eulji Medical Center, Eulji University School of Medicine, Uijeongbu; \\ ${ }^{2}$ Department of Otorhinolaryngology-Head and Neck Surgery, Samsung Medical Center, Sungkyunkwan University School of Medicine, Seoul,
}

Korea

Objectives. The aim of this study was to compare the long-term oncologic outcomes of sentinel lymph node biopsy (SLNB) versus elective neck dissection (END) in clinically node-negative (cN0) tongue cancer.

Methods. This was a retrospective cohort study of patients with cN0 tongue cancer from a single institution, including 91 patients in the SLNB group and 120 patients in the END group.

Results. The overall recurrence rate showed no significant difference between the two groups. The regional control rate was also comparable between the two groups $(P=0.490)$. The 5-year recurrence-free survival $(\mathrm{RFS})$ was slightly better in the SLNB group than in the END group $(P=0.427)$. The 5-year overall survival $(\mathrm{OS})$ rate was $89.9 \%$ in the SLNB group versus $91.9 \%$ in the END group $(P=0.737)$. In a propensity-matched subgroup analysis, the type of neck management did not affect RFS or OS.

Conclusion. SLNB showed non-inferior oncologic outcomes compared to END in patients with cN0 tongue squamous cell carcinoma.

Keywords. Recurrence; Sentinel Lymph Node; Neck Dissection

\section{INTRODUCTION}

Since the presence of lymph node metastases is one of the most critical factors in the survival and recurrence rate of oral cancer,

\footnotetext{
- Received December 1, 2020

Revised March 14, 2021

Accepted March 26, 2021

- Corresponding author: Man Ki Chung

Department of Otorhinolaryngology-Head and Neck Surgery, Samsung

Medical Center, Sungkyunkwan University School of Medicine,

81 Irwon-ro, Gangnam-gu, Seoul 06351, Korea

Tel: +82-2-3410-3577, Fax: +82-2-3410-6987

E-mail: chungmk@skku.edu

- Co-Corresponding author: Chung-Hwan Baek

Department of Otorhinolaryngology-Head and Neck Surgery, Samsung

Medical Center, Sungkyunkwan University School of Medicine,

81 Irwon-ro, Gangnam-gu, Seoul 06351, Korea

Tel: +82-2-3410-3579, Fax: +82-2-3410-6987

E-mail: chbaek@skku.edu
}

accurate detection and proper management of nodal disease are essential [1,2]. It is well known that the occult metastasis rate in clinically node-negative ( $\mathrm{cN} 0)$ patients with oral cancer ranges from $21 \%$ to $35 \%$ and that occult metastasis is challenging to detect, even with a thorough physical examination and imaging studies $[3,4]$. The proper neck management of $\mathrm{cN} 0$ oral cancer patients has long been controversial. A recent randomized controlled trial compared elective neck dissection (END) versus therapeutic neck dissection as treatment options for $\mathrm{cN} 0$ oral cancer [5]. The patients who underwent END were reported to have an improved 3-year overall survival (OS) rate over therapeutic neck dissection ( $80 \%$ vs. $67.5 \%$ ), with an occult metastasis rate of $26.5 \%$. Based on this landmark study, END was accepted as the standard treatment for $\mathrm{cN} 0$ oral cancer patients. However, debate continues regarding the necessity of surgical procedures for the substantial proportion of patients (over half) who do not have any metastatic disease [4].

Copyright $@ 2022$ by Korean Society of Otorhinolaryngology-Head and Neck Surgery

This is an open-access article distributed under the terms of the Creative Commons Attribution Non-Commercial License (https://creativecommons.org/licenses/by-nc/4.0)

which permits unrestricted non-commercial use, distribution, and reproduction in any medium, provided the original work is properly cited. 
Recently, sentinel lymph node biopsy (SLNB) is gaining popularity because many studies have reported evidence supporting the proposal that SLNB is a useful staging procedure to detect occult nodal metastasis accurately, theoretically helping threequarters of patients with $\mathrm{cN} 0$ oral squamous cell carcinoma to avoid unnecessary surgery [6-15]. According to other prospective multicenter trials, the negative predictive value of SLNB is reported to be around $95 \%$, depending on the subsite of the tumor $[6,10]$. False-negative or false-omission rates were $9.8 \%-$ $14 \%$, although these rates should be maintained under $5 \%$ [16]. Despite recognizing the acceptable diagnostic accuracy of SLNB for detecting occult nodal metastasis, there are still hurdles for its widespread application in clinical practice by many head and neck surgeons [17]. One of the hurdles is the scarcity of research directly comparing oncologic outcomes of SLNB with END to justify routine SLNB application $[18,19]$.

This study aimed to evaluate the oncologic safety of SLNB in the management of $\mathrm{cN} 0$ oral tongue cancer in direct comparison with END. In a retrospective cohort study of a single institution, we compared the regional control rate, recurrence-free survival (RFS), and OS according to whether patients underwent SLNB or END. A propensity score analysis was performed to achieve a more balanced comparison, matching the resection margin to reduce the retrospective design's potential bias.

\section{MATERIALS AND METHODS}

\section{Patients}

The study was approved by Institutional Review Board of Samsung Medical Center (SMC IRB 2020-11-166). Retrospective cohort enrollments and medical records review were exempt from the patient's informed consent. We enrolled patients who had tongue cancer with $\mathrm{cN} 0$ early tongue squamous cell carcinoma treated surgically in Samsung Medical Center between January 1995 and December 2018 in the study. Patients with advanced $\mathrm{T}$ stage (T3 or T4) or those who had undergone other tongue cancer treatments before surgery were excluded. To dis-

\section{H I G H L I I G H T S}

- From a single-institutional database, long-term oncologic outcomes were compared between patients with $\mathrm{cN} 0$ tongue cancer according to whether sentinel lymph node biopsy (SLNB) or elective neck dissection (END) was performed.

- The regional control rate, 5-year recurrence-free survival, and overall survival were comparable between the SLNB and END groups, and the findings were reproducible in a propensitymatched subgroup analysis.

- SLNB was an acceptable alternative to END in cN0 tongue cancer. tinguish the recurrence and failure of positive node detection, cases with short follow up within 6 months were also eliminated. Physical evaluation, computed tomography, magnetic resonance imaging, and positron emission tomography were evaluated as a preoperative workup for clinical nodal staging. If necessary, ultrasonography-guided fine-needle aspiration cytology was also performed.

In our institution, the treatment of choice for the patients with cN0 tongue cancer has been surgical resection of the primary tumor with END since the establishment of the head and neck cancer center in January 1995. As described in the previous study, SLNB was introduced in our institution after 2002, starting with the validation phase to test the procedure's feasibility and safety [20]. After 2007, an SLNB alone trial was applied in eligible patients, designed to do the subsequent neck dissection only in cases with positive sentinel lymph node(s) in the pathologic examination. Since 2007, cN0 tongue cancer patients who consented to the SLNB procedure have undergone SLNB alone trial, while the rest received conventional END for the cN0 neck management. Adjuvant treatments, including radiation with/without chemotherapy, were performed on patients with a high-risk feature in the final pathologic report, such as a positive node or advanced T stage.

\section{Neck management}

We used END to remove the ipsilateral lymph nodes located in neck-level I-III or IV, depending on preoperative imaging studies' findings. The SLNB procedure was performed, as described previously [21]. The radioactive tracer, technetium $99 \mathrm{~m}$ prepared with tin colloid (Amerscan Hepatate II; Nycomed Amersham Health, London, UK), was injected in the submucosal layer around the circumference of the primary tumor $(5-6 \mathrm{mCi}$ in $0.6 \mathrm{~mL})$. Dynamic lymphoscintigraphy was done in the anterior and lateral views before surgery. During operation, all radioactive lymph nodes were identified with a Navigator GPS hand-held gamma probe (Tyco Healthcare, Mansfield, MA, USA) and were given thorough transcervical dissection for the removal of the primary tumor. If the sentinel lymph node was not detected by lymphoscintigraphy preoperatively or by gamma probe during surgery, END was performed. In our study, none of the cases showed the failure of sentinel node identification. The mean number of sentinel nodes dissected during surgery was 2.84 (range, 1-7).

Sentinel lymph nodes were divided into multiple equal levels of approximately 2-mm thickness through their longest axes and separately frozen with an Optimal Cutting Temperature compound. One section for each block was evaluated using frozen section analysis with hematoxylin and eosin (H\&E) staining. After the frozen section diagnosis, the frozen tissue blocks were melted, fixed in $10 \%$ neutral buffered formalin, and embedded in paraffin. These paraffin blocks were serially sectioned at $250 \mu \mathrm{m}$ (step-serial sectioning), and six sections from each level within the block were H\&E stained and examined for possible metas- 
tasis. We did further immunohistochemical analysis for cytokeratin (AE1/AE3) to reveal any undetected micrometastasis if the node was free from tumors. If sentinel lymph node(s) showed a metastatic tumor in frozen biopsy or permanent pathology, subsequent therapeutic neck dissection was done. Decisions on the postoperative, adjuvant treatment were made by the multidisciplinary tumor board, assessing all the clinicopathological factors. Recurrence was defined as a new diagnostic lesion in the neck detected at least 6 months after the end of treatment. The lesion developed within 6 months was regarded as a failure of neck dissection.

\section{Statistical analysis}

The two groups' clinical characteristics were evaluated, including age, sex, clinical and pathologic stage based on the American Joint Committee on Cancer (AJCC) 7th edition, resection margin, depth of invasion, and presence of perineural invasion/lymphovascular invasion.

Patient characteristics were compared for equality by the MannWhitney $U$-test for continuous variables and chi-square test or Fisher's exact test for categorical data. Propensity score matching analysis allowed the adjustment of baseline patients' characteristics between the END and the SLNB groups (one-to-one matching based on propensity scores). We used a binary logistic regression model to develop a propensity score for each patient. The depth of invasion, a critical factor for recurrence and survival, was included in the propensity score model. Matching was done using calipers of a width of 0.5 of the standard deviation of the propensity score's logit. The standardized difference between unmatched and matched groups was 0.17. A doubly robust method was performed to correct the remained bias.

RFS and OS were analyzed by Kaplan-Meier statistics. We used the log-rank test to compare survival rates between the two groups and multivariate analysis to find the correlation between survival and each clinicopathological variable. Statistical packages of $R$ and SAS ver. 9.4 (SAS Institute, Cary, NC, USA) were used for statistical analyses, and a $P<0.05$ was considered statistically significant.

\section{RESULTS}

A total of 211 patients were enrolled in the study, with 91 patients in the SLNB group and 120 in the END group. Patients' characteristics are described in Table 1 . The age and sex distribution were similar between the two groups. The SLNB group had more patients diagnosed with cT1 disease than the END group (SLNB vs. END, $80.2 \%$ vs. $54.2 \%$; $P<0.001$ ).

In the postoperative pathologic data, the END group showed worse features than did the SLNB group, with a greater depth of invasion (6.96 mm vs. $5.29 \mathrm{~mm}, P=0.009)$ and a more frequent presence of lymphovascular invasion $(15.8 \%$ vs. $4.4 \%, P=0.013)$. Pathologic $\mathrm{T}$ staging revealed a difference between the two groups, with a higher frequency of T2 disease in the END group than in the SLNB group (47.5\% vs. $18.7 \%$ according to the AJCC 7 th edition, $67.5 \%$ vs. $40.7 \%$ according to the AJCC 8th edition, both $P<0.001)$. When patients were reclassified using the AJCC 8th edition, 24 patients in the END group and 20 patients in the SLNB group were upstaged from pT1 to pT2. The overall proportion maintained the same statistical tendency regardless of whether the seventh or 8th edition was used. The resection margin in the SLNB group was greater than that in the END group (5.66 mm vs. $4.63 \mathrm{~mm}, P=0.001$ ). The mean follow-up was 47.2 months, with 38.2 months in the END group and 58.9 months

Table 1. Patients' characteristics

\begin{tabular}{|c|c|c|c|c|}
\hline Variable & Total patients $(n=211)$ & END $(n=120)$ & SLNB $(n=91)$ & $P$-value \\
\hline \multicolumn{5}{|l|}{ Demographic data } \\
\hline Sex & & & & 0.414 \\
\hline Male & $129(56.9)$ & $70(58.3)$ & $59(64.8)$ & \\
\hline Female & $82(38.9)$ & $50(41.7)$ & $32(35.2)$ & \\
\hline Age (yr) & $53.12 \pm 13.63$ & $54.52 \pm 13.34$ & $51.27 \pm 13.86$ & 0.086 \\
\hline \multicolumn{5}{|l|}{ Preoperative data } \\
\hline cT1:T2 & $138: 73$ & $65: 55$ & $73: 18$ & $<0.001$ \\
\hline \multicolumn{5}{|l|}{ Postoperative data } \\
\hline pT1:T2:T3:T4 (AJCC 7th ed) & $127: 74: 6: 4$ & $57: 57: 5: 1$ & $70: 17: 1: 3$ & $<0.001$ \\
\hline pT1:T2:T3:T4 (AJCC 8th ed) & $83: 118: 6: 4$ & $33: 81: 5: 1$ & $50: 37: 1: 3$ & $<0.001$ \\
\hline Resection margin (mm) & $5.07 \pm 2.34$ & $4.63 \pm 2.00$ & $5.66 \pm 2.63$ & 0.001 \\
\hline Depth of invasion (mm) & $6.24 \pm 4.63$ & $6.96 \pm 4.80$ & $5.29 \pm 4.25$ & 0.009 \\
\hline Lymphovascular invasion & $23(10.9)$ & $19(15.8)$ & $4(4.4)$ & 0.013 \\
\hline Perineural invasion & $29(13.7)$ & $21(17.5)$ & $8(8.8)$ & 0.106 \\
\hline Pathologically positive lymph node & $25(11.8)$ & $19(15.9)$ & $6(6.6)$ & 0.040 \\
\hline Follow-up period (mo) & $47.2(5.8-193.9)$ & $38.2(5.8-193.9)$ & $58.9(7.9-160.0)$ & $<0.001$ \\
\hline
\end{tabular}

Values are presented as number (\%), mean \pm standard deviation, or mean (range).

END, elective neck dissection; SNLB, sentinel lymph node biopsy; AJCC, American Joint Committee on Cancer. 
in the SLNB group.

The total number of $\mathrm{pN}+$ patients after $\mathrm{cN} 0$ neck management was $25(11.8 \%)$. This proportion was significantly higher in the END group (15.8\%) than in the SLNB group (6.6\%) $(P=0.040)$ (Fig. 1). Of the patients with pN+ disease in the END group, $89.5 \%$ received adjuvant treatment (postoperative radiotherapy with or without chemotherapy), whereas $66.7 \%$ of $\mathrm{pN}+$ patients in the SLNB group underwent adjuvant treatment.

Recurrence was reported in 32 of 211 patients (15.1\%). The rate of regional recurrence was $10.0 \%$ (21/211). Eleven patients in the SLNB group (12.0\%) and 10 patients in the ENB group $(8.3 \%)$ showed regional recurrence $(P=0.490)$. The duration until recurrence was similar between the two groups (SLNB vs. END, 592.26 vs. 606.19 days; range, 23-4,214 days). Failure of neck dissection, as shown by the rates of regional metastasis within 6 months, was similar in both groups: 4.4\% (4/91) in the SLNB group and 3.3\% (4/120) in the END group $(P=0.73)$. Most patients with recurrence $(28 / 32,87.5 \%)$ underwent salvage surgery. Only four patients received radiation or chemotherapy for recurrence due to refusal of surgery and distant metastasis. At the last follow-up, 18 patients were alive without disease, three patients were alive with disease, and 11 patients had died from the disease. The regional control rate showed no statistically significant difference (SLNB vs. END, $87.9 \%$ vs. $91.7 \%, P=0.490$ ).

RFS was slightly better in the SLNB group; however, the difference was not statistically significant $(84.6 \%$ in the SLNB group vs. $79.5 \%$ in the END group, $P=0.427$ ) (Fig. 2A). The KaplanMeier survival estimate of the two groups was compared, and the 5 -year OS was $89.9 \%$ in the SLNB group versus $91.9 \%$ in the END group ( $P=0.737$ ) (Fig. 2B). Cox regression analysis was done to identify the prognostic factors for RFS and OS. For RFS, sex and the depth of invasion tended toward significance, but not

\section{cNO neck management $\begin{aligned} & \text { Pathologic } \\ & \text { outcomes }\end{aligned}$ Postoperative adjuvant treatment \& follow up Regional control rate}

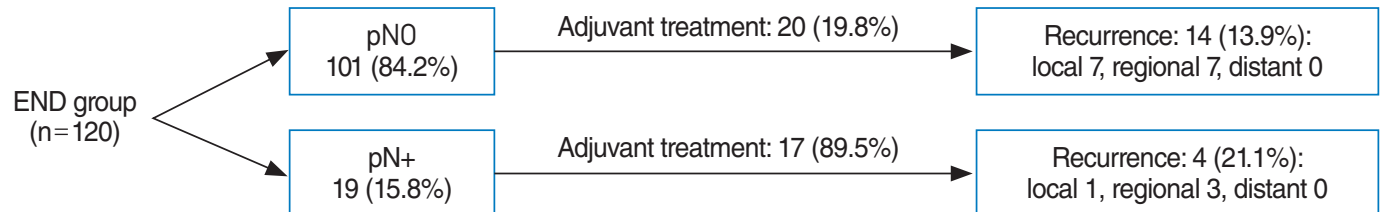

$91.7 \%(110 / 120)$ ocal 1 , regional 3 , distant 0

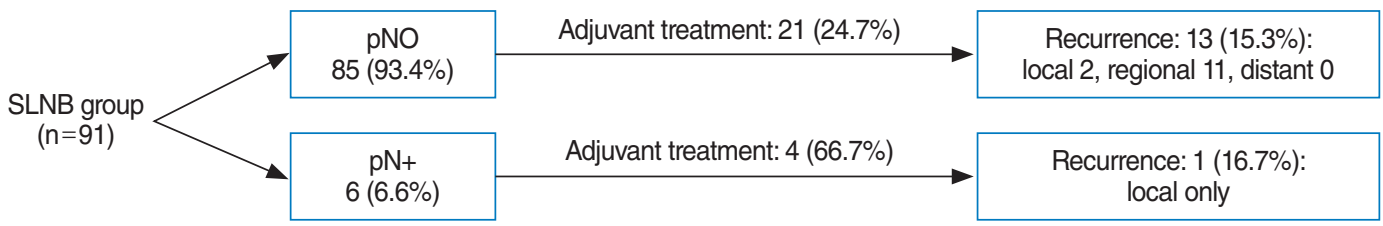

Fig. 1. Clinical course of the elective neck dissection (END) group and sentinel lymph node biopsy (SLNB) group.

A

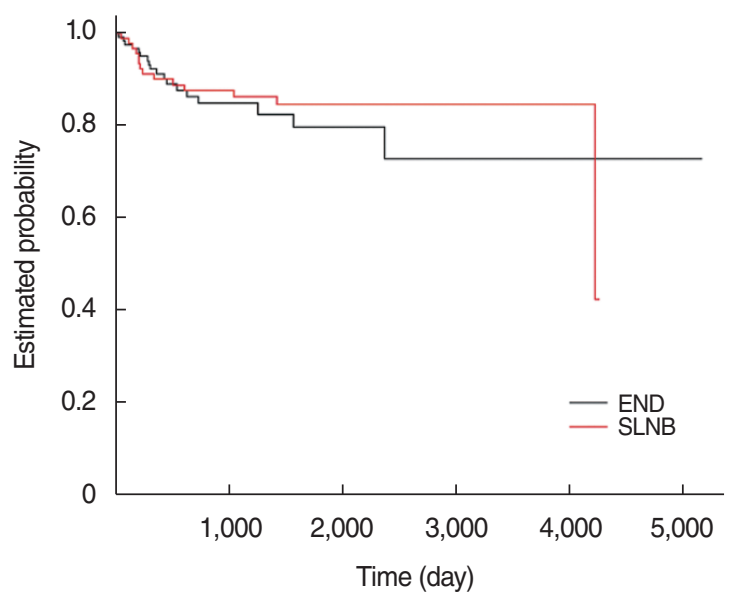

B

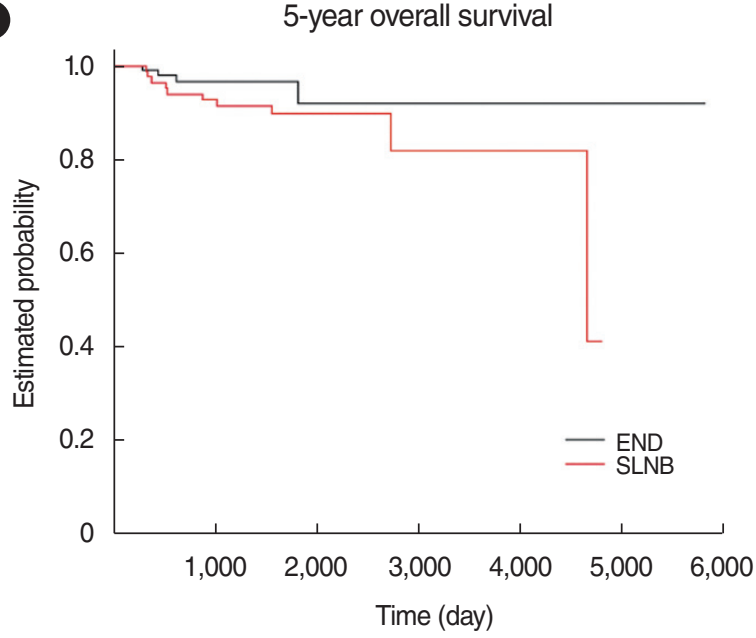

Fig. 2. Kaplan-Meier estimates of the sentinel lymph node biopsy (SLNB) group and elective neck dissection (END) group. (A) Five-year recurrence-free survival: END 79.5\% vs. SLNB 84.6\% ( $P=0.427)$. (B) Five-year overall survival: END 91.9\% vs. SLNB 89.9\% (P=0.737). 
Table 2. Cox regression analysis of risk factors affecting recurrence-free survival

\begin{tabular}{|c|c|c|c|c|c|c|}
\hline \multirow{2}{*}{ Variable } & \multicolumn{3}{|c|}{ Univariate analysis } & \multicolumn{3}{|c|}{ Multivariate analysis } \\
\hline & $\mathrm{HR}$ & $95 \% \mathrm{Cl}$ & $P$-value & $\mathrm{HR}$ & $95 \% \mathrm{Cl}$ & $P$-value \\
\hline \multicolumn{7}{|l|}{ Sex } \\
\hline Male & 1 & & & & & \\
\hline Female & 0.291 & $0.108-0.788$ & 0.015 & 0.332 & $0.136-0.814$ & 0.016 \\
\hline Age (continuous) & 0.991 & $0.962-1.021$ & 0.544 & & & \\
\hline \multicolumn{7}{|l|}{ T stage $^{\text {a) }}$} \\
\hline $\mathrm{T} 1$ & 1 & & & & & \\
\hline $\mathrm{T} 2$ & 3.045 & $1.158-8.004$ & 0.024 & & & \\
\hline T3 & 3.116 & $0.285-34.133$ & 0.352 & & & \\
\hline $\mathrm{T} 4$ & 0.000 & 0.000 & 0.999 & & & \\
\hline \multicolumn{7}{|l|}{$\mathrm{N}_{\text {stage }}{ }^{\mathrm{a})}$} \\
\hline No & 1 & & & & & \\
\hline $\mathrm{N}+$ & 1.126 & $0.329-3.855$ & 0.850 & & & \\
\hline Resection margin (continuous) & 0.972 & $0.807-1.170$ & 0.764 & & & \\
\hline Depth of invasion (continuous) & 1.078 & $0.974-1.192$ & 0.146 & 1.070 & $1.002-1.143$ & 0.043 \\
\hline \multicolumn{7}{|l|}{ LVI } \\
\hline Absent & 1 & & & & & \\
\hline Present & 0.000 & 0.000 & 0.998 & & & \\
\hline \multicolumn{7}{|l|}{$\mathrm{PNI}$} \\
\hline Absent & 1 & & & & & \\
\hline Present & 0.673 & $0.156-2.907$ & 0.596 & & & \\
\hline \multicolumn{7}{|l|}{ Type of neck management } \\
\hline END & 1 & & & & & \\
\hline SLNB & 1.368 & $0.551-3.394$ & 0.499 & 0.914 & $0.448-1.865$ & 0.804 \\
\hline
\end{tabular}

$\mathrm{HR}$, hazard ratio; Cl, confidence interval; LVI, lymphovascular invasion; PNI, perineural invasion; END, elective neck dissection; SNLB, sentinel lymph node biopsy.

a)American Joint Committee on Cancer (AJCC) 7th ed.

Table 3. Cox regression analysis of risk factors affecting overall survival

\begin{tabular}{|c|c|c|c|c|c|c|}
\hline \multirow{2}{*}{ Variable } & \multicolumn{3}{|c|}{ Univariate analysis } & \multicolumn{3}{|c|}{ Multivariate analysis } \\
\hline & $\mathrm{HR}$ & $95 \% \mathrm{Cl}$ & $P$-value & $\mathrm{HR}$ & $95 \% \mathrm{Cl}$ & $P$-value \\
\hline \multicolumn{7}{|l|}{ Sex } \\
\hline Male & 1 & & & & & \\
\hline Female & 0.262 & $0.048-1.420$ & 0.120 & 0.279 & $0.061-1.267$ & 0.098 \\
\hline Age (continuous) & 1.018 & $0.970-1.068$ & 0.474 & 1.046 & $1.002-1.092$ & 0.038 \\
\hline \multicolumn{7}{|l|}{ T stage $^{a)}$} \\
\hline $\mathrm{T} 1$ & 1 & & & & & \\
\hline $\mathrm{T} 2$ & 4.493 & 0.968-20.858 & 0.055 & & & \\
\hline T3 & 0.000 & 0.000 & 0.999 & & & \\
\hline T4 & 68.498 & $2.787-1,683.532$ & 0.010 & & & \\
\hline \multicolumn{7}{|l|}{ N stage ${ }^{a)}$} \\
\hline NO & 1 & & & & & \\
\hline $\mathrm{N}+$ & 2.403 & $0.462-12.494$ & 0.297 & & & \\
\hline Resection margin (continuous) & 1.090 & $0.850-1.397$ & 0.498 & & & \\
\hline Depth of invasion (continuous) & 1.101 & $0.952-1.274$ & 0.194 & 1.114 & $1.013-1.225$ & 0.026 \\
\hline \multicolumn{7}{|l|}{ LVI } \\
\hline Absent & 1 & & & & & \\
\hline Present & 0.000 & 0.000 & 0.998 & & & \\
\hline \multicolumn{7}{|l|}{ PNI } \\
\hline Absent & 1 & & & & & \\
\hline Present & 0.489 & $0.044-5.390$ & 0.559 & & & \\
\hline \multicolumn{7}{|l|}{ Type of neck management } \\
\hline END & 1 & & & & & \\
\hline SLNB & 5.374 & $1.160-24.893$ & 0.032 & 2.754 & $0.844-8.983$ & 0.093 \\
\hline
\end{tabular}

HR, hazard ratio; Cl, confidence interval; LVI, lymphovascular invasion; PNI, perineural invasion; END, elective neck dissection; SNLB, sentinel lymph node biopsy.

a)American Joint Committee on Cancer (AJCC) 7th ed. 
Table 4. Multivariate analysis of risk factors affecting recurrence and survival after propensity matching

\begin{tabular}{|c|c|c|c|c|c|c|}
\hline \multirow{2}{*}{ Variable } & \multicolumn{3}{|c|}{ Recurrence-free survival } & \multicolumn{3}{|c|}{ Overall survival } \\
\hline & $\mathrm{HR}$ & $95 \% \mathrm{Cl}$ & $P$-value & $\mathrm{HR}$ & $95 \% \mathrm{Cl}$ & $P$-value \\
\hline Resection margin & 0.909 & $0.758-1.090$ & 0.305 & 0.943 & $0.736-1.208$ & 0.943 \\
\hline Depth of invasion & 1.063 & $0.977-1.158$ & 0.157 & 1.113 & $0.990-1.252$ & 0.074 \\
\hline \multicolumn{7}{|c|}{ Type of neck management } \\
\hline END & 1 & & & & & \\
\hline SLNB & 0.814 & $0.356-1.860$ & 0.626 & 2.144 & $0.563-8.172$ & 0.264 \\
\hline
\end{tabular}

$\mathrm{HR}$, hazard ratio; $\mathrm{Cl}$, confidence interval; END, elective neck dissection; SNLB, sentinel lymph node biopsy.

(4)

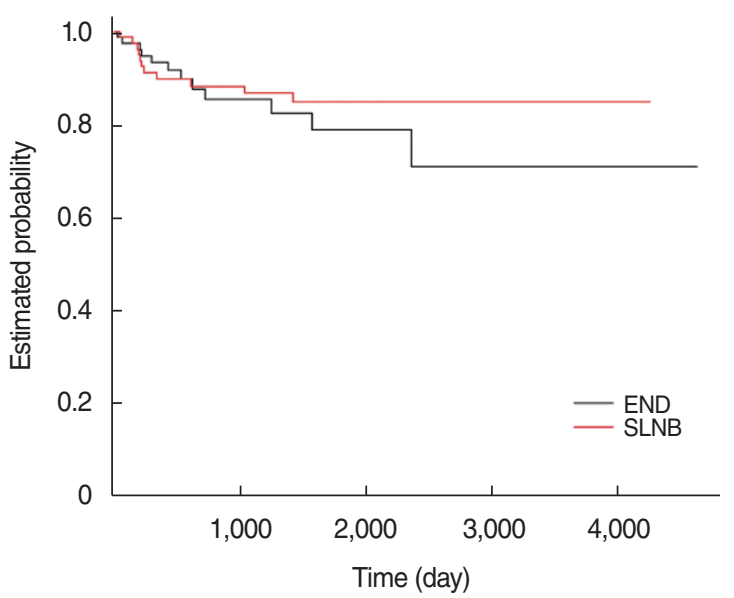

B

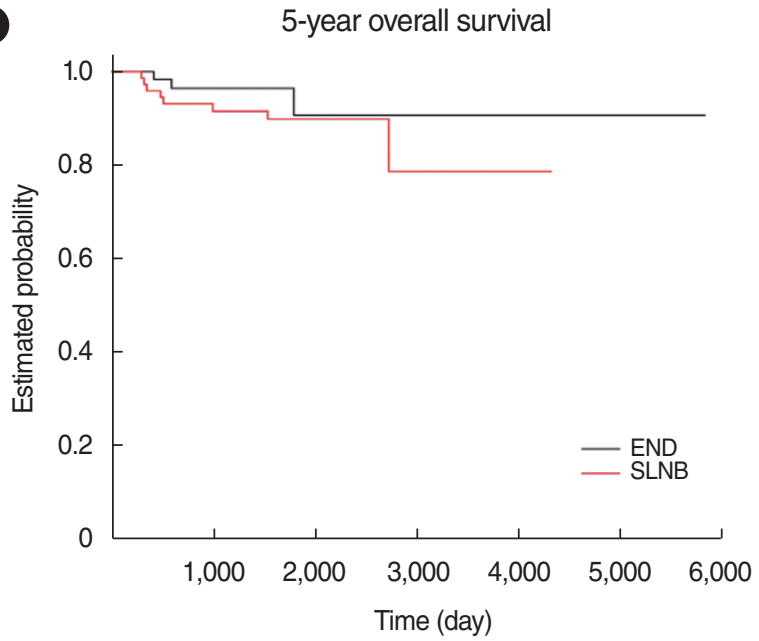

Fig. 3. Kaplan-Meier estimates of the sentinel lymph node biopsy (SLNB) subgroup and elective neck dissection (END) subgroup after propensity-matching analysis. (A) Five-year recurrence-free survival: END 78.7\% vs. SLNB 84.8\% ( $P=0.423)$. (B) Five-year overall survival: END $90.6 \%$ vs. SLNB $89.7 \%(P=0.899)$.

the type of neck management (Table 2). Deeper invasion was a significant prognostic factor for worse OS (hazard ratio, 1.114) (Table 3).

We used propensity score matching to adjust for the uneven distribution of depth of invasion, which is a critical factor for the primary oncologic outcomes. As a result, subgroups of 79 patients from each group were generated (Supplementary Table 1). An analysis of the two groups using the Cox regression method showed that the neck management type did not influence the prognosis or recurrence (Table 4). According to the doubly-robust analysis conducted herein, the type of neck management had no evident effect on RFS or OS (Fig. 3).

\section{DISCUSSION}

This study compared the long-term oncologic outcomes between END and SLNB in cN0 tongue cancer patients with a mean follow-up of 47.2 months, using a single institution's retrospective cohort. We found that the two groups' regional control rates were comparably excellent $(91.7 \%$ vs. $87.9 \%, P=0.490)$, without a statistically significant difference. The 5 -year OS and RFS also did not significantly differ between the two groups (SLNB group vs. END group: OS, $89.9 \%$ vs. $91.9 \%$, log-rank $P=0.737$; RFS, $84.6 \%$ vs. $79.5 \%$, log-rank $P=0.427$ ). Furthermore, postoperative clinical progress was described and compared in detail between the two groups. This finding could give head and neck surgeons informative data for introducing the SNLB procedure in their clinical practice.

The study's oncologic results are similar to those of a previous study that retrospectively compared 30 SLNB patients and 52 END patients with oral tongue squamous cell carcinoma [19]. According to the previous study, the regional recurrence rate was $13.3 \%(4 / 30)$ in the SLNB group and 9.6\% (5/52) in the END group, and both RFS and OS were comparable between the two groups, without statistically significant differences (10-year RFS, $72.3 \%$ vs. $73.3 \%$; 10 -year OS, $43.3 \%$ vs. $44.2 \%$ ). These findings suggest that SLNB could be a substitute modality for END with no influence on survival.

A commonly cited advantage of SLNB is that patients with no sign of metastases in sentinel lymph nodes could avoid neck dissection. Individualized treatment could reduce both morbidity and cost [7]. Moreover, based on a detailed analysis of the postoperative course, the number of patients who received adju- 
vant treatment (SLNB 27.5\% vs. END 30.8\%, $P=0.65$ ) was similar in the two groups, indicating that the choice of neck treatment had no adverse effect on prognosis (Fig. 1). Both groups' recurrence rates showed similar outcomes, even though pN0 patients in the SLNB group did not receive additional therapeutic neck dissection or adjuvant treatment. Approximately a third of $\mathrm{pN}+$ patients in the SLNB group did not receive postoperative adjuvant treatment, and they did not experience any recurrence during follow-up.

To maintain an acceptable oncologic outcome in an SLNBalone trial, the false-negative rate must be as low as 5\% (pN0 sentinel lymph node progression within 2 years) [16]. There were eight regional recurrent cases $(3.3 \%$ and $4.4 \%)$ within 6 months in each group, which would be considered failures of neck management or false-negatives. Salvage operations were done in all eight patients, of whom five patients survived without any evidence of disease, one patient was still alive with the disease at the last follow-up, and two patients died from the disease. This finding is comparable to that of a previous study reporting longterm follow-up outcomes and the nodal failure pattern of SLNB in oral squamous cell carcinoma [22]. Among 11 patients with regional recurrence in the SLNB group, three cases of contralateral nodal recurrence and one ipsilateral nodal recurrence were found at almost 10 years after the SLNB. As highlighted in our previous report about the SLNB-alone trial, a stringent followup protocol with salvage treatment is critical for maintaining acceptable oncologic outcomes [20]. Close follow-up is mandatory for positive SLNB patients, who are at higher risk of nodal recurrence and worse prognosis than negative SLNB patients.

In a recent study, a large number of patients with stage I-II oral squamous cell carcinoma (240 patients who underwent SLNB and 8,088 patients who underwent END) were retrieved and analyzed from the National Cancer Data Base of the United States [18]. The study's outcomes were similar to the present study in that 3-year OS was equivalent between the two groups (82.0\% after SLNB vs. $77.5 \%$ after END, $P=0.40)$. Interestingly, SLNB showed a significantly shorter length of postoperative hospital stay compared to END and an absolute difference in the 30 -day mortality rate ( $0 \%$ in SLNB vs. $0.7 \%$ in END). Despite all these advantages, it was found that the SLNB procedure was rarely used for stage I-II oral cancer, warranting a multicenter randomized control trial directly comparing SLNB and END to produce level-I evidence for SLNB in early oral cancer [18].

This study is affected by the common drawbacks of all studies using a retrospective cohort, including selection bias. Firstly, key pathologic features, such as the surgical resection margin and the depth of invasion, were not equally distributed between the SLNB group and the END group. To overcome the uneven distribution of adverse pathologic factors, we performed a propensity score matching analysis based on the resection margin to generate subgroups of 79 patients from each group. Secondly, we did not investigate other controversial issues related to each procedure, for example, cost-effectiveness, surgery-related complications, or quality-of-life comparisons.

According to this retrospective cohort analysis, the SLNB did not have worse oncologic outcomes than END in patients with cN0 tongue squamous cell carcinoma. Considering that many patients could avoid unnecessary invasive treatment after nodal staging by SLNB, this procedure is highly recommended in clinical practice. A watchful follow-up protocol, as well as a potential plan for salvage management, is warranted to obtain excellent outcomes for a long-term period.

\section{CONFLICT OF INTEREST}

No potential conflict of interest relevant to this article was reported.

\section{ORCID}

Woori Park https://orcid.org/0000-0001-9032-6685

Hokyung Jin https://orcid.org/0000-0002-7278-0320

Yujin Heo https://orcid.org/0000-0001-9577-6802

Han-Sin Jeong https://orcid.org/0000-0003-4652-0573

Young-Ik Son https://orcid.org/0000-0003-0570-6953

Man Ki Chung https://orcid.org/0000-0002-1435-7786

Chung-Hwan Baek https://orcid.org/0000-0003-4430-5546

\section{AUTHOR CONTRIBUTIONS}

Conceptualization: MKC, CHB. Data curation: YIS, HSJ, MKC, CHB. Formal analysis:WP, MKC, CHB. Methodology:WP, MKC. Project administration: WP, MKC, CHB. Visualization: WP, HJ, YH.Writing-original draft: WP, HJ, YH, MKC. Writing-review \& editing: WP, MKC, CHB.

\section{SUPPLEMENTARY MATERIALS}

Supplementary materials can be found via https://doi.org/10. 21053/ceo.2020.02411.

\section{REFERENCES}

1. Rogers SN, Brown JS, Woolgar JA, Lowe D, Magennis P, Shaw RJ, et al. Survival following primary surgery for oral cancer. Oral Oncol. 2009 Mar;45(3):201-11.

2. Woolgar JA, Rogers S, West CR, Errington RD, Brown JS, Vaughan ED. Survival and patterns of recurrence in 200 oral cancer patients treated by radical surgery and neck dissection. Oral Oncol. 1999 May;35(3):257-65. 
3. Teichgraeber JF, Clairmont AA. The incidence of occult metastases for cancer of the oral tongue and floor of the mouth: treatment rationale. Head Neck Surg. 1984 Oct;7(1):15-21.

4. Kligerman J, Lima RA, Soares JR, Prado L, Dias FL, Freitas EQ, et al. Supraomohyoid neck dissection in the treatment of T1/T2 squamous cell carcinoma of oral cavity. Am J Surg. 1994 Nov;168(5): 391-4.

5. D’Cruz AK, Vaish R, Kapre N, Dandekar M, Gupta S, Hawaldar R, et al. Elective versus therapeutic neck dissection in node-negative oral cancer. N Engl J Med. 2015 Aug;373(6):521-9.

6. Schilling C, Stoeckli SJ, Haerle SK, Broglie MA, Huber GF, Sorensen JA, et al. Sentinel European Node Trial (SENT): 3-year results of sentinel node biopsy in oral cancer. Eur J Cancer. 2015 Dec;51(18): 2777-84.

7. Schilling C, Shaw R, Schache A, McMahon J, Chegini S, Kerawala C, et al. Sentinel lymph node biopsy for oral squamous cell carcinoma. Where are we now? Br J Oral Maxillofac Surg. 2017 Oct;55(8):75762.

8. Samant S. Sentinel node biopsy as an alternative to elective neck dissection for staging of early oral carcinoma. Head Neck. 2014 Feb; 36(2):241-6.

9. Flach GB, Bloemena E, KlopWM, van Es RJ, Schepman KP, Hoekstra OS, et al. Sentinel lymph node biopsy in clinically N0T1-T2 staged oral cancer: the Dutch multicenter trial. Oral Oncol. 2014 Oct;50(10): 1020-4.

10. Civantos FJ, Zitsch RP, Schuller DE, Agrawal A, Smith RB, Nason R, et al. Sentinel lymph node biopsy accurately stages the regional lymph nodes forT1-T2 oral squamous cell carcinomas: results of a prospective multi-institutional trial. J Clin Oncol. 2010 Mar;28(8):1395-400.

11. Wu JX, Hanson M, Shaha AR. Sentinel node biopsy for cancer of the oral cavity. J Surg Oncol. 2019 Aug;120(2):99-100.

12. Krishnamurthy A. Current status and future perspectives of sentinel lymph node biopsy in oral cancers. Indian J Dent Res. 2017 May-Jun; 28(3):239-40.

13. Hernando J, Villarreal P, Alvarez-Marcos F, Gallego L, Garcia-Consuegra L, Junquera L. Comparison of related complications: sentinel node biopsy versus elective neck dissection. Int J Oral Maxillofac
Surg. 2014 Nov;43(11):1307-12.

14. Den Toom IJ, Heuveling DA, Flach GB, van Weert S, Karagozoglu $\mathrm{KH}$, van Schie A, et al. Sentinel node biopsy for early-stage oral cavity cancer: theVU University Medical Center experience. Head Neck. 2015 Apr;37(4):573-8.

15. Stoeckli SJ, Alkureishi LW, Ross GL. Sentinel node biopsy for early oral and oropharyngeal squamous cell carcinoma. Eur Arch Otorhinolaryngol. 2009 Jun;266(6):787-93.

16. Garrel R, Poissonnet G, Temam S, Dolivet G, Fakhry N, de Raucourt D. Review of sentinel node procedure in $\mathrm{cN} 0$ head and neck squamous cell carcinomas: guidelines from the French evaluation cooperative subgroup of GETTEC. Eur Ann Otorhinolaryngol Head Neck Dis. 2017 Apr;134(2):89-93.

17. Seim NB,Wright CL,Agrawal A. Contemporary use of sentinel lymph node biopsy in the head and neck. World J Otorhinolaryngol Head Neck Surg. 2016 Jul;2(2):117-25.

18. Cramer JD, Sridharan S, Ferris RL, Duvvuri U, Samant S. Sentinel lymph node biopsy versus elective neck dissection for stage I to II oral cavity cancer. Laryngoscope. 2019 Jan;129(1):162-9.

19. Fan SF, Zeng ZY, Peng HW, Guo ZM, Wang SL, Zhang Q. Sentinel lymph node biopsy versus elective neck dissection in patients with cT1-2N0 oral tongue squamous cell carcinoma. Oral Surg Oral Med Oral Pathol Oral Radiol. 2014 Feb;117(2):186-90.

20. Chung MK, Lee GJ, Choi N, Cho JK, Jeong HS, Baek CH. Comparative study of sentinel lymph node biopsy in clinically N0 oral tongue squamous cell carcinoma: long-term oncologic outcomes between validation and application phases. Oral Oncol. 2015 Oct;51(10): 914-20.

21. Jeong HS, Baek CH, Son YI, Cho DY, Chung MK, Min JY, et al. Sentinel lymph node radiolocalization with $99 \mathrm{mTc}$ filtered tin colloid in clinically node-negative squamous cell carcinomas of the oral cavity. J Korean Med Sci. 2006 Oct;21(5):865-70.

22. Moya-Plana A, Auperin A, Guerlain J, Gorphe P, Casiraghi O, Mamelle $G$, et al. Sentinel node biopsy in early oral squamous cell carcinomas: long-term follow-up and nodal failure analysis. Oral Oncol. 2018 Jul;82:187-94. 